\title{
Temperature Distribution Prediction in Control Cooling Process with Recurrent Neural Network for Variable-Velocity Hot Rolling Strips
}

Dong Chen ( $\square$ neu_chendong@163.com )

Northeastern University https://orcid.org/0000-0003-2809-4486

Rui Zhang

Northeastern University

Zhenlei Li

Northeastern University

Yunjie Li

Northeastern University

Guo Yuan

Northeastern University

\section{Research Article}

Keywords: Hot rolling strip, variable-velocity rolling, temperature distribution prediction, recurrent neural network

Posted Date: January 20th, 2022

DOI: https://doi.org/10.21203/rs.3.rs-1257684/v1

License: (c) (1) This work is licensed under a Creative Commons Attribution 4.0 International License.

Read Full License 


\title{
Temperature Distribution Prediction in Control Cooling Process with Recurrent
}

\section{Neural Network for Variable-velocity Hot Rolling Strips}

\author{
Dong Chen, Rui Zhang, Zhenlei Li, Yunjie Li, Guo Yuan* \\ ${ }^{1}$ State Key Laboratory of Rolling and Automation, Northeastern University, P. O. Box. 105, No. 11, Lane 3, \\ Wenhua Road, HePing District Shenyang 110819, Liaoning, China. \\ *Corresponding author: Guo Yuan, E-mail: yuanguoral@ sina.com; Tel: +86 24 83688543; Fax: +86 24 \\ 23906472
}

\begin{abstract}
Control cooling is essential method for microstructure and mechanical properties control in hot rolling strip making. It is vital to realize high precises temperature distribution prediction and control in cooling process to ensure the industrial production. In this paper, a traditional mechanism model based on finite-difference method and combining with online cycle velocity calculation strategy was introduced as one of estimating temperature distribution baseline method. However, considering calculation time, variable-velocity rolling makes it difficult to rapidly realize temperature and water distribution modifying of each segment in cooling zone. Herein, a temperature distribution prediction method based on recurrent neural network was proposed, instead of only final cooling temperature prediction. And temperature distribution prediction performance of model with different recurrent cell and time steps were evaluated. The results indicated that the proposed model could realize temperature distribution prediction and the model based on bi-LSTM and 48 timesteps has the highest determination coefficient value of 0.976 and lowest root mean square error of 8.03 and mean absolute error of 5.7. Furthermore, compared with baseline model, the proposed model retained lower computational cost, making it applicable in industrial application by providing real-time temperature distribution prediction.
\end{abstract}

Key words: Hot rolling strip, variable-velocity rolling, temperature distribution prediction, recurrent neural network; 


\section{Introduction}

As an important fundamental raw material, hot rolling strips are widely used in industries, automobile, shipbuilding, and other fields. In hot strips making, control cooling process on run out table is vital for steel product pursuing excellent and stable performance[1]. After finishing rolling, the strip is cooled by water from a start cooling temperature to target temperature with a given cooling rate before coiled, which promotes obtaining more fine grain size and improving strengthening and toughness[2,3]. In control cooling process, high precise temperature distribution prediction and cooling path control are key factor for ensuring the mechanical property[4].

In cooling process, as the strip deliveries from finishing mill to down coiler, water distribution must be adjusted with variable rolling velocity to meet target coil temperature (CT). Water cooling process is complexly affected by many factors, mainly including strip temperature, water flow, strip thickness, chemical composition and strip velocity, etc.[5,6] Once the strip enters cooling zone, strip thickness, width, and composition have little difference in strip length direction, start cooling temperature and velocity are the main factors influencing temperature control. Currently, to increase the productivity and decrease heat loss on delay of transfer bar, finishing rolling process always adopts the variable-velocity rolling strategy in hot rolling strips making, which leads strips velocity varying in a large scale[7]. Meanwhile, the adjustment of rolling velocity is also used to control finishing mill delivery temperature (FDT, herein also is start cooling temperature), by which the rolling acceleration/deceleration and velocity are dynamically changed to meet the aim FDT and significantly influence coil temperature control accuracy[8]. Under variablevelocity rolling, water distribution expressed as open/closed cooling headers and header flow is the only adjustment method to guarantee target coil temperature. Therefore, precise water distribution calculation based on accuracy velocity perdition is crucial for cooling path and temperature distribution control in 
cooling process[9].

To build a high-accuracy temperature distribution prediction and control model, many scholars have been paying attention to related research. So far, three main kinds of models have been developed and applied. Index model is an important scheme to deal with convective heat transfer, considering convective between cooling water and strip surface as the main heat exchange in cooling process $[10,11]$. However, due to simplifications and ignoring temperature gradient in strip thickness, the application effect of index model is not good, especial for heavy thickness plates. Another kind of model is statistical model, which was built relying on statistical regularity[12,13]. In statistical modelling, by fully considering physical mechanism of cooling process, the main influence parameters of cooling process were considered and determined by statistical regressive methods[14-17]. Further, to improve the control precision, the influence parameters were classified to categories based on steel grades and thicknesses of strip or other boundary conditions. Then, each category of strips has a series of model parameters. However, the temperature control accuracy of this method was limited by the number of categories. Furthermore, some advanced methods were used to improve the control accuracy, such as fuzzy control and model prediction control[12,18,19]. While the advanced methods in control cooling are greatly rely on the statistical model or index model. The third model is built by finite difference method (FDM) and heat transfer theory, which divides strip and time into nodes and finite number of steps to solve differential equations by approximate derivatives. Thence, the spatial and sequential temperature distribution of strip could be obtained[20,21]. During modeling, for surface node, the heat transfer form is radiation and convection heat transfer, while the heat transfer form is heat conduction for inner nodes. In control cooling process, the temperature gradient in thickness direction is fully considered in FDM, consequently, control accuracy was obvious improved, especially for heavy thickness strip. At present, the most used method is combining with FDM 
and statistical method, the former is used for temperature prediction model building, and latter is used for seeking optimal model parameters.

As above-mentioned, strip velocity significantly influences cooling process. To realize high precise prediction of velocity in cooling process, time-velocity-distance (TVD) method and real-time modify are commonly used methods. Li proposed a velocity-controlled strategy for each strip element based on TVD[22]. And an online cyclic modification and calculation strategy is designed to minimize the temperature fluctuation in variable velocity rolling. However, consume time of modification and calculation for each segment exceed the requirement of online system. Based on this, Li proposed an improved method that could realize online monitor and control of cooling temperature by modifying at setpoint and decrease the computation time[20]. However, due to the irregular changes of hot rolling, the more times of temperature modifying is benefit for the temperature control. Thence, a new temperature prediction method which could quickly realize prediction and repeatedly match the more times velocity modifying is needed.

Although, the introduced temperature prediction methods and models are still accepted and widely used due to practicality and stability, but it is difficult to further improve control level and satisfied the requirement mentioned above. Therefore, some model based on machine learning was developed, and show prospect of high control accuracy.

As one of the well-known instance-based learning methods, KNN has been widely applied in many fields, which is also generally used in control cooling process. Zheng adopted an new approach to build model for accelerated cooling process by IIR-KNN[23]. Based on the $k$ selected similar case, locally linear reconstruction is applied to determine best output parameters for current plate. Zhang proposed a variable scale grid model for temperature self-adaptive control, which builds a multi-dimension space system and 
each dimension is determined by effect of each factor on heat transfer[24]. Every plate can find a corresponding point in space, according to its own process conditions to correct temperature calculation. Neural network is another one of most popular algorithms used in steel industry, which has stronger nonlinearity and capability of adaptive information processing. Some researchers have successfully adopted ANNs to predict heat transfer coefficient, temperature or water flow[25-27]. Valentina introduced an ANN to find correlations between model parameters and process variables[28]. Xing developed a hybrid intelligent identification model by combining the RBF neural networks, CBR and fuzzy logic reasoning, which can make a great contribution in improving the coil temperature precision by prediction precision of correct identification[29]. However, in above mentioned, variable strip velocity and temperature distribution in cooling process were ignored. They only take constant velocity and final cooling temperature into consideration. Obviously, in order to keep mechanical performance fluctuation within a narrow range, those are not suit for the variable-velocity rolling and cannot realize temperature distribution prediction.

In view of above problems, considering the ability of dealing with sequence, a novel temperature prediction model based on recurrent neural network was proposed and constructed, which could accurately predict temperature distribution under variable-velocity rolling. To improve the precise and robust, the Pauta criterion and isolate forest algorithm were used to preprocess the industrial data collected from control cooling process. In view of particular of water distribution feature, the feature process method was introduced. The loss function was modified to improve training efficiency and accuracy of the model. In addition, the recurrent cell and length of input sequence were tested to determined optimal recurrent neural network model. 


\section{Control cooling process on run out table of hot rolling strips}

\subsection{Description of cooling process}

The schematic diagram Fig. 1 shows control cooling process of hot rolling strips on run out table. During cooling process, the strip will be sent into run-out table after being delivered from finishing mill, and then it will be cooled in following long cooling zone and finally coiled by down coiler. The cooling zone is made up by ultra-fast cooling (UFC) and laminar cooling. UFC system lies between finishing mill and laminar cooling, contains 40 top headers and 40 bottom headers, divided into 4 groups. UFC header flow and pressure can be adjusted stepless for different cooling ability with range of $50 \sim 300 \mathrm{~m}^{3} / \mathrm{h}$ and $0.3 \sim 1.0 \mathrm{MPa}$, respectively. Laminar cooling is consisted of 72 U-type top headers and 72 straight bottom headers, divided into 16 groups. The foremost 14 groups are main cooling section and the 1ast two groups are vernier cooling for feedback control. In cooling process, the number of cooling headers and the water flow of each header are taken as control variables to adjust temperature distribution of the strip.

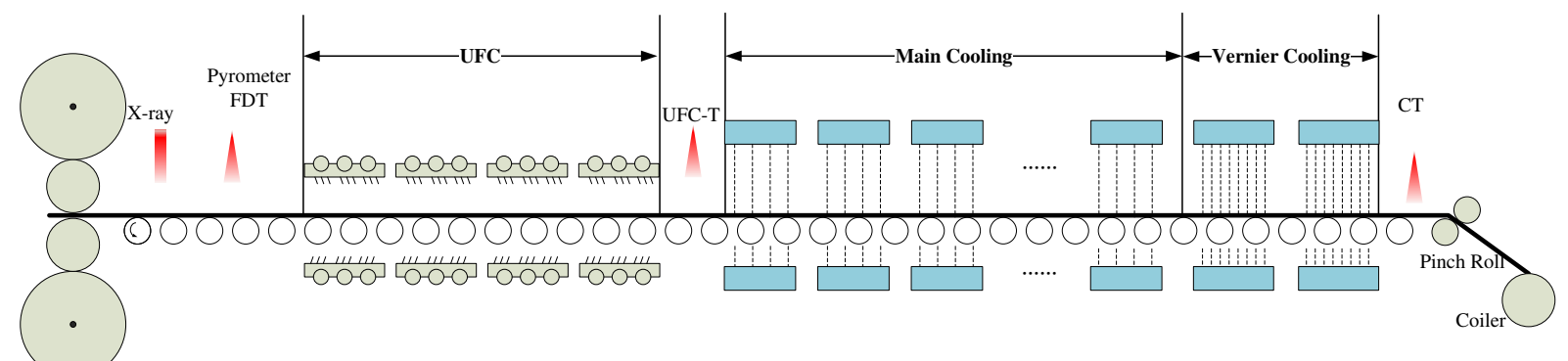

Fig. 1 Schematic diagram of cooling system on run out table

\subsection{Thermal dynamic model}

Considering heat transfer in thickness direction is much higher than width direction and rolling direction for high rolling velocity and uniform cooling capability of cooling headers, heat dissipation along length and width directions could be ignored. As well, heat latent of phase transaction could be contained in correction of specific heat. The heat transfer equation is simply expressed as Eq. (1): 


$$
\rho c_{p} \frac{\partial t}{\partial \tau}-\frac{\partial}{\partial x}\left[\lambda \frac{\partial t}{\partial x}\right]=0
$$

with boundary conditions on top and bottom surfaces can be described in form like Newton's convection Eq. (2), and equivalent to water cooling and air cooling:

$$
\pm \lambda \frac{\partial t}{\partial x}=h\left(t-T_{m}\right)
$$

where, $\rho$ is density of strip steels in $\mathrm{kg} / \mathrm{m}^{3}, c_{\mathrm{p}}$ is specific heat in $\mathrm{J} /(\mathrm{kg} \cdot \mathrm{K}), \lambda$ is coefficient of thermal conductivity in $\mathrm{W} /(\mathrm{m} \cdot \mathrm{K}), t$ is temperature of strip steels in $\mathrm{K}, \tau$ is heat transfer time in $\mathrm{s}, x$ is thickness direction, $T_{\mathrm{m}}$ is cooling water temperature in $\mathrm{K}$.

Finite difference method was used to model internal heat transfer through conduction. And finite difference calculations maintained the temperature of each node as piece moves from FDT pyrometers to CT pyrometers. As shown in Fig. 2, the half thickness of strip was researched. With boundary condition, the node in thickness Eq.(1) can be expressed in explicit difference form as Eq.(3):

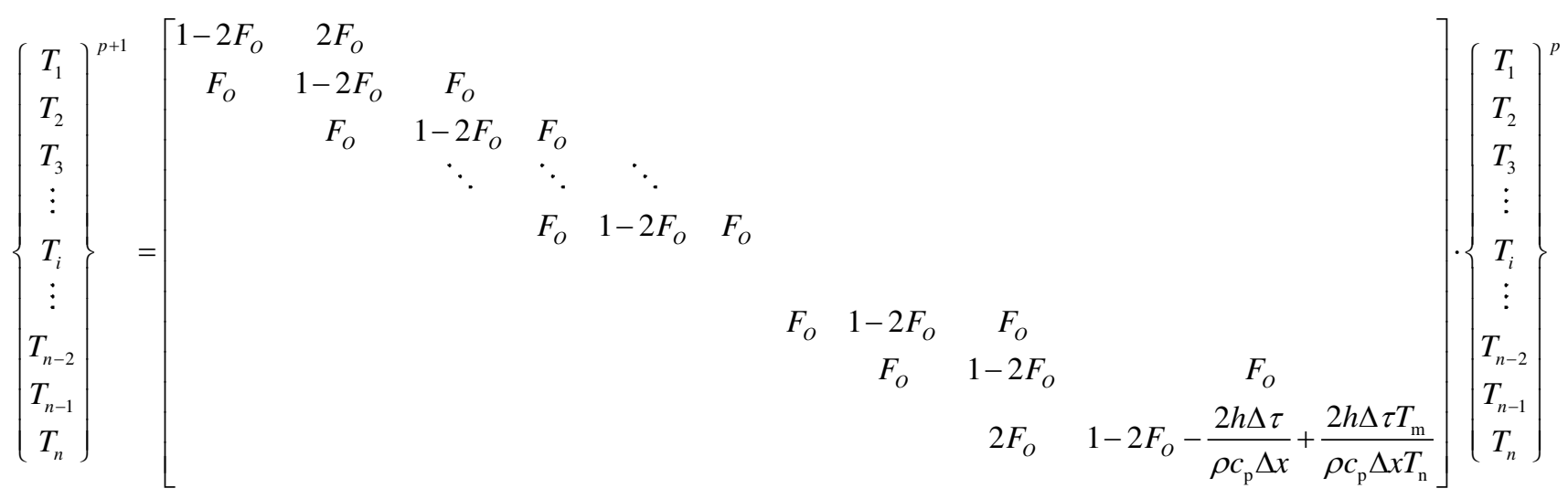

where,

$$
F o=\frac{\lambda \Delta \tau}{\rho c_{\mathrm{p}}(\Delta x)^{2}}
$$

For air cooling zone boundary conditions, the equivalent heat transfer coefficient is given by Eq. (5):

$$
h_{a}=\varepsilon K\left(t+T_{a}\right)\left(t^{2}+T_{a}^{2}\right)
$$


where, $\varepsilon$ is radiation of strip in $\mathrm{W}, K$ is Stephen-Boltzmann constant in $\mathrm{J} / \mathrm{K}$.

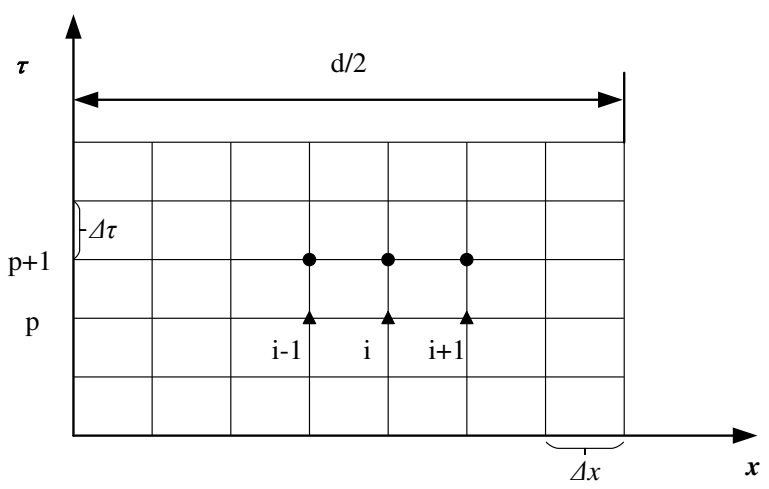

Fig. 2 One-dimensional region discretization

For water cooling zone boundary conditions, the main factors which affect water convection heat transfer are water flow and temperature, strip velocity and thickness. In addition, water pressure in UFC process is also a key factor for water convection. So heat transfer coefficient of water cooling in UFC system and laminar cooling can be calculated as follows Eq. (6) and Eq. (7), respectively.

$$
\begin{gathered}
h_{w}=\alpha A_{1} \cdot \frac{Q}{B L}(h)^{A_{2}} \cdot \exp \left(-A_{3}\left(t-T_{w}\right)\right)\left(\frac{T_{w}}{T_{o}}\right)^{A_{4}}\left(\frac{P}{P_{o}}\right)^{A_{s}}\left(\frac{V}{V_{o}}\right)^{A_{\sigma}} \\
h_{w}=\alpha A_{1} \cdot \frac{Q}{B L}(h)^{A_{2}} \cdot \exp \left(-A_{3}\left(t-T_{w}\right)\right)\left(\frac{T_{w}}{T_{o}}\right)^{A_{4}}\left(\frac{V}{V_{o}}\right)^{A_{6}}
\end{gathered}
$$

where, $\alpha$ is correction coefficient of strip width, $A_{1}-A_{6}$ are model coefficients, $\mathrm{Q}$ is summation water flow in $\mathrm{m}^{3} / \mathrm{h}, T_{w}$ is measured water temperature in $\mathrm{K}, P$ is measured cooling water pressure in MPa, $V$ is measured velocity of strip in $\mathrm{m} / \mathrm{s}, T_{o}$ is reference cooling water temperature in $\mathrm{K}, P_{o}$ is reference water pressure in $\mathrm{MPa}, V_{o}$ is based velocity in $\mathrm{m} / \mathrm{s}, B$ is width of cooling bank in $\mathrm{m}, \mathrm{L}$ is effective cooling length in $\mathrm{m}$.

Based on above equations, the through thickness temperature field can be obtained, once the parameters in heat transfer coefficient equations were determined. As can be seen in Eq.(6) (8), water flow, strip temperature, water temperature and pressure were easier to obtain, the difficulty was to get accurate strip 
velocity in variable-velocity rolling.

\section{The online cycle velocity calculation strategy}

In rolling process, strip velocity combining with inter-stand cooling are regulated to keep the measured strip temperature meet target FDT. Generally, strip goes through low velocity threading, first acceleration, high acceleration and tail out deceleration as shown in Fig. 3, the typical time velocity and distance curve (TVD curve). Meanwhile, rolling acceleration or deceleration will be changed, when the measured FDT exceeds allowable tolerance. Due to variable-velocity rolling, water distribution should be dynamic adjustment to guarantee final coil temperature. Thus, accuracy velocity predict for strip is basis for temperature prediction and water distribution control. To realize high accuracy velocity prediction, an online cycle velocity calculation algorithm was introduced.

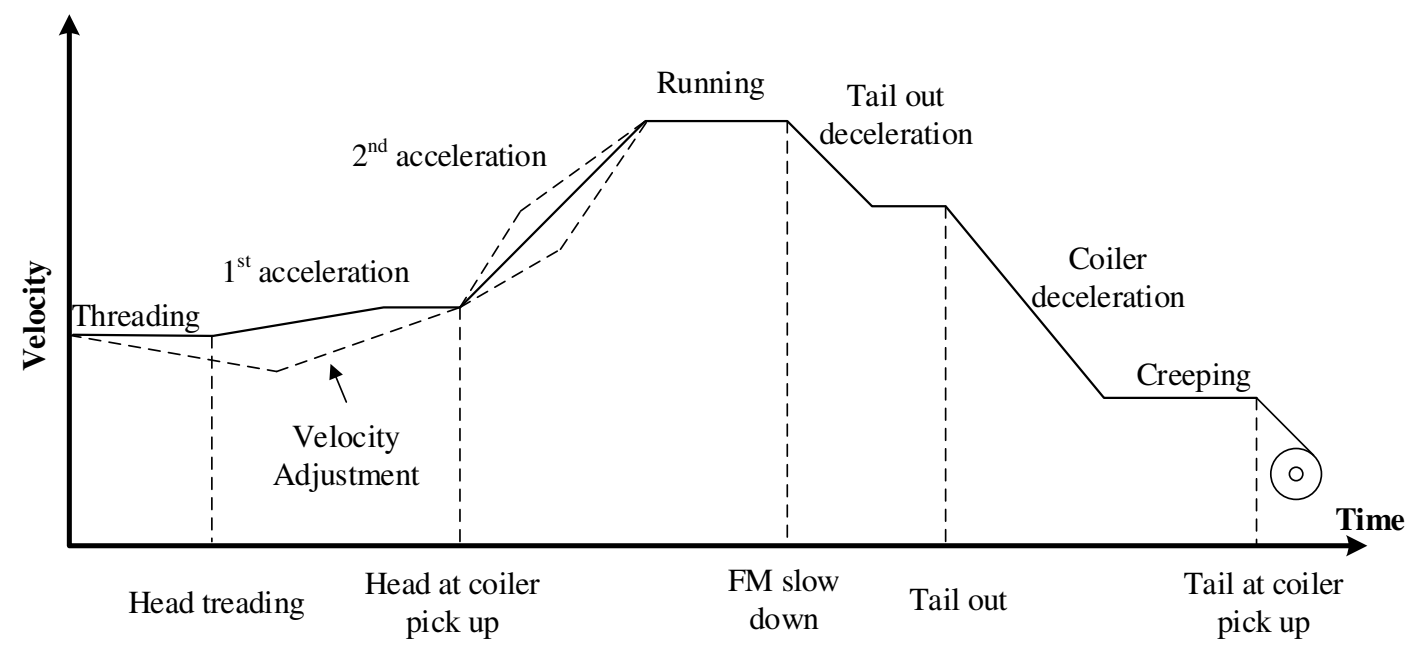

Fig. 3 The typical strip time-velocity-distance curve

To achieve high accuracy temperature control in whole strip length, the strip is divided into numbers of fixed length segments, and each segment was separately controlled. Velocity curve and water-cooling time of each segment passing through run out table needs to be predicted before the segment enters the cooling section. Generally, in different rolling stage, strip velocity change has certain characteristics. Then, entire length of strip was divided into five parts based on characteristic of TVD curve: low velocity threading, 
low acceleration before strip coiled, high acceleration to max running speed, tail out deceleration and the remaining length uncoiled, named of $S_{1} \sim S_{5}$ part for convenience. The on-line cycle velocity calculation algorithm is shown in Fig. 4.

To begin with, each part length $l_{i}$ and velocity $v_{i}$ of $S_{i}(i$ values from 1 to 5$)$ was calculated with finishing rolling preset threading velocity, acceleration/deceleration according to kinematics formula Eq. (8). In this way, the overall TVD curve of strip was determined. In addition, running velocity of the segment in cooling section was calculated based on the overall TVD curve. Herein, running velocity of each segment was discerned as entrance and exit velocity of $V_{i, j}$ and $V_{i, j}^{\prime}$ at $j^{\text {th }}$ micro cooling section. Furthermore, prediction velocity was utilized to calculate the temperature distribution on run out table and water distribution would be adjusted to satisfy the target temperature. Once a new segment arrives FDT, velocity calculation algorithm will be trigged. Based on real-time rolling velocity and acceleration/deceleration, the above three steps will be repeated. After re-calculated, velocity of all segments in cooling zone would be modified.

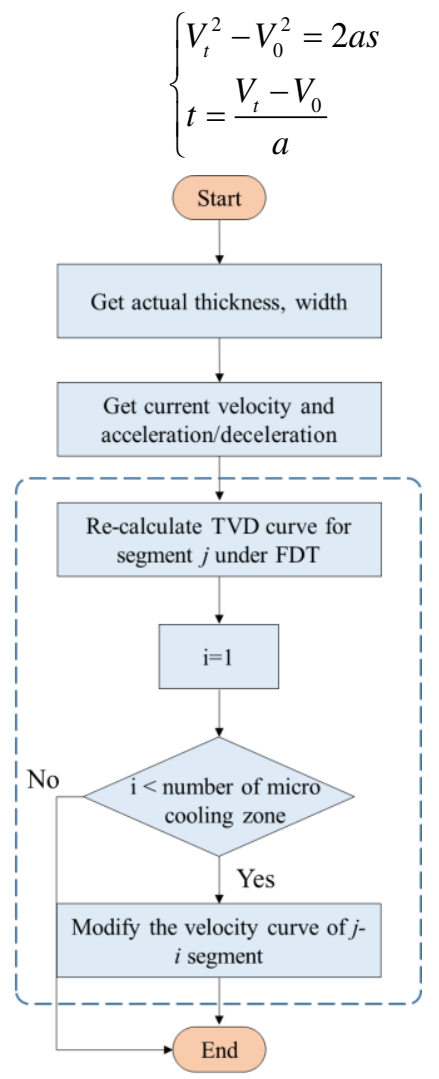


Fig. 4 Work flow of online cycle velocity calculation algorithm

The following figures in Fig. 5 are calculation velocity and actual velocity curve of $4.25 \mathrm{~mm}$ SPA-H and 13.75mm Q355B, respectively. The profile shows the above-mentioned velocity calculation algorithm can accurately predict the strip velocity.
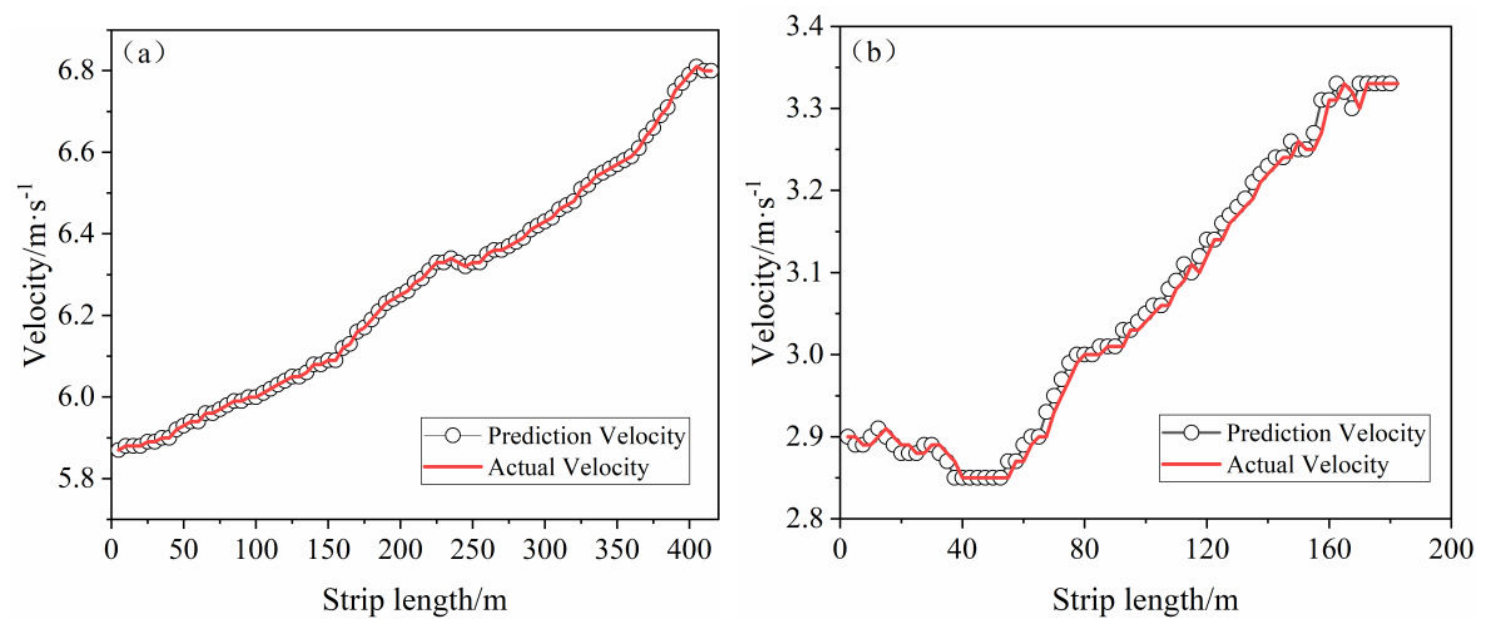

Fig. 5 Profile of actual velocity and calculation velocity

(a) $4.25 \mathrm{~mm}$ SPA-H; (b) $13.75 \mathrm{~mm}$ Q355B

\section{Deep Learning Approach for Temperature Distribution Prediction}

\subsection{Problem existing}

With above thermal dynamic model and on-line cycle velocity calculation strategy, it would be possible to achieve desired cooling process temperature controlling by re-calculation of temperature distribution and modifying water distribution, simultaneously. The more times of TVD curves updating, the higher accuracy velocity and temperature prediction. However, it would occupy excessive computation resources of temperature re-calculation and modifying, which could not meet requirements of online model. Despite of improved method were researched, it is all try to reduce times of velocity and temperature update which is not benefit to temperature control in cooling process. Therefore, it is necessary to build a less-computation model which also could achieve variable-velocity rolling temperature prediction to enhance capability of dynamic control. 


\subsection{Fundamental of Recurrent neural network}

Recurrent neural network (RNN) is a type of recursive neural network, which takes sequence data as input, performs recursion in evolution direction of sequence, and RNN was developed to solve issue of the information forgotten in tradition feed-forward and feedback neural networks[30]. As shown in Fig. 6, given an input sequence $\boldsymbol{X}=\left(x_{1}, x_{2}, \ldots ., x_{t}\right)$, a standard RNN computes the hidden vector sequence $\boldsymbol{S}=\left(s_{1}, s_{2}, \ldots ., s_{t}\right)$ and output vector sequence $\boldsymbol{Y}=\left(y_{1}, y_{2}, \ldots ., y_{t}\right)$ by iterating following equations from $t=1$ to $t$ as following:
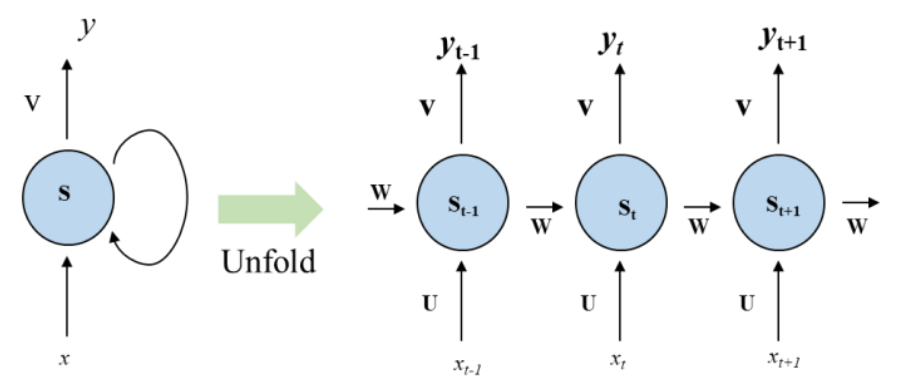

Fig. 6 Structure of recurrent neural network

$$
\begin{gathered}
y_{t}=g\left(V * S_{t}\right) \\
s_{t}=f\left(U * s_{t}+W * s_{t-1}\right)
\end{gathered}
$$

where, $\boldsymbol{V}$ terms denote weight matrices of hidden-output layer, $\boldsymbol{U}$ terms is input-hidden layer weight matrices. $\boldsymbol{W}$ terms express hidden to hidden step weight matrices. $\boldsymbol{g}$ and $\boldsymbol{f}$ are function activation function, and $\boldsymbol{f}$ is usually an element wise application of a sigmoid function. It can be seen from Fig. $\mathbf{6}$, the values of hidden layer not only depend on current input $x_{t}$, but also on previous hidden layers. In other words, RNNs has memory of the previous input content. Due to the 'memory', the RNNs and its evolutions have performed well in sequence tasks and were widely used in text recognition, speech recognition, real-time translation, and image identification[31-33].

Subsequently, long short-term memory (LSTM) unit $[34,35]$ and its simple version gated recurrent unit 
(GRU) [36,37]are proposed to overcome vanishing gradient and capture long-term dependency. As can be seen in Fig. 7, compared with standard RNN, LSTM and GRU have more complex structure. In standard RNN, repeating module only have a very simple structure, tanh layer. While in LSTM, the repeating module has a different structure, except for tanh layer, there are also three different gates, forget gate $f_{t}$, input gate $\boldsymbol{i}_{t}$ and output gate $\boldsymbol{o}_{t}$. To achieve the long-term dependency, at each time step, the cell state $c_{t}$ is slowly updated to new unit, which is determined by $f_{t}$ and $i_{t}$. The $f_{t}$ controls the memory with sigmoid activation function based on input vector $\boldsymbol{x}_{\boldsymbol{t}}$, and prior hidden state $\boldsymbol{h}_{\boldsymbol{t}-1}$, while $\boldsymbol{i}_{\boldsymbol{t}}$ controls remembering current information. Finally, hidden state $\boldsymbol{h}_{t}$ is calculated using $\boldsymbol{o}_{\boldsymbol{t}}$ and $\boldsymbol{c}_{\boldsymbol{t}}$ with tanh activation. Furthermore, GRU is simplify from LSTM to decrease a large amount of learning parameters. As shown in Fig. 7 (c), different with LSTM, $\boldsymbol{h}_{t}$ is determined by an update gate $z_{t}$ and a reset gate $\boldsymbol{r}_{t}$, while no longer a cell state exists in the unit. The former controls how much information from the previous hidden state will carry over to the current one, whilst the latter decides whether the previous hidden state is forgettable[37].
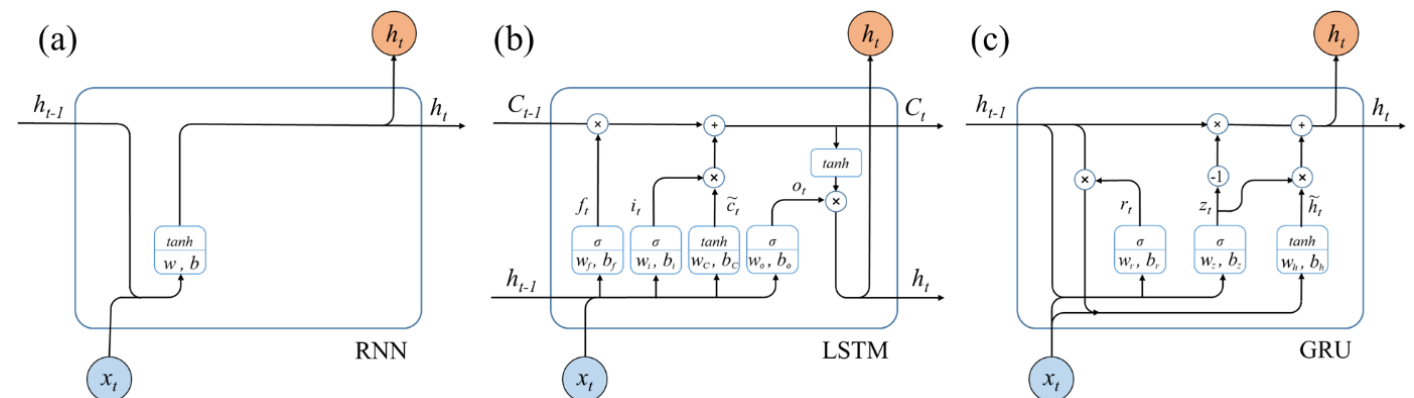

Fig. 7 Schematic diagram of recurrent units: (a) standard recurrent neural network, (b) operation of LSTM unit and, (c) operation of GRU unit

\subsection{Temperature distribution prediction based on recurrent neural networks}

As above section 3 mentioned, to realize high precise temperature control of entire strip length, the strip is divided into number of segments, and each segment is separately controlled. Correspondingly, cooling zone is also divided into several micro cooling zone, same length with segment. In cooling process, each 
segment passes through all micro cooling zone in sequence from FDT pyrometer to CT pyrometer, with temperature drop increasing, strip temperature finally meets the target value. Obviously, strip temperature calculation in each micro cooling zone has obvious feature of sequence data. Thence, RNN algorithm can be adopt to prediction cooling temperature in variable-velocity process based on existing models. Taking one temperature calculation in micro cooling zone as one timestep, the illustration of the proposed model is shown in Fig. 8. Except for basic parameter of strip, such as thickness, width which is almost not change, strip velocity and water distribution in each micro cooling zone are divided into input sequential values, and temperature distribution sequence is output. It should be noted: $T, V$ and $F$ in shown in following Fig. 8 mean temperature, velocity, and water distribution in each cooling zone.

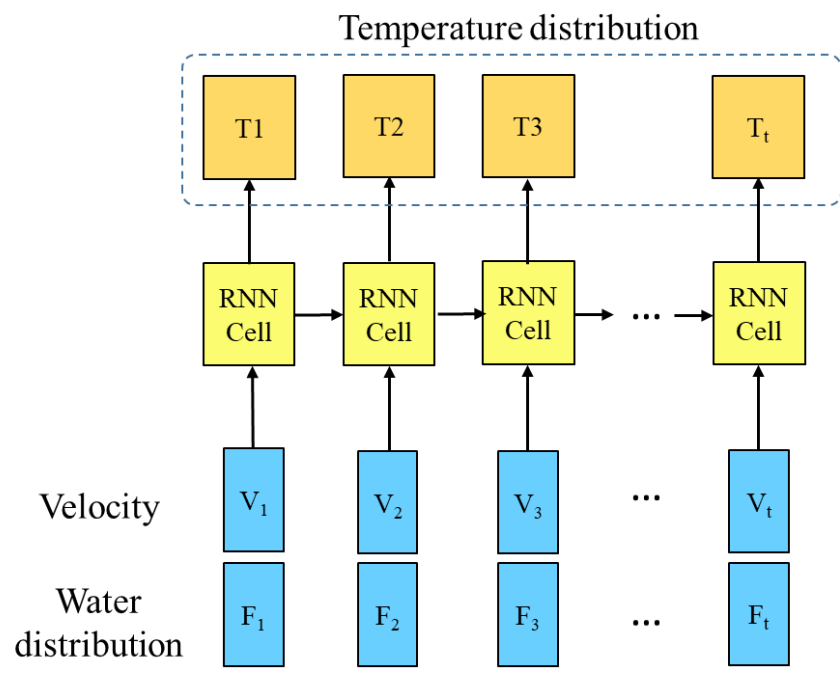

Parameters in each micro cooling zone

Fig. 8 Proposed RNN model for temperature distribution prediction

In hot rolling strip line, automation system records almost all of process parameters in database or production logs, mainly includes real-time data and model setup data. Two months of 12998 coils production data relevant to control cooling process on run out table have been collected. First, due to the unstable measuring of pyrometer, data of each strip head end and tail end is removed. Then, the remained data are randomly sampled and combined to obtain 99000 experiment strip segments data. These samples 
are used to develop temperature distribution prediction model based on RNN. 19 key variables including temperature distribution data are selected for neural network modelling. For the reason of medium temperature in cooling zone are hardly to be measured, temperature distribution except for initial temperature FDT and final cooling temperature CT measured, other temperature distribution values was padding with tradition model re-calculation values based on measured temperature data. The descriptions of dataset are shown in Table 1 and Fig. 9. In Fig.9, fractal dimension visualization diagrams of experiment data show distribution of significant variables and output data. The enormous amount of data proves the developed model will have a strong robustness with dataset. In particular, total flow here is used for abnormal detection.

Table 1 Description of parameter of input and output variable for RNN model

\begin{tabular}{|c|c|c|c|c|c|c|}
\hline No. & Parameter & Description & Unit & Range & Mean & Std \\
\hline 1 & $t$ & Strip thickness & $\mathrm{mm}$ & {$[1.6,20]$} & 4.201 & 2.216 \\
\hline 2 & $W$ & Strip width & $\mathrm{mm}$ & {$[836,2075]$} & 1391.406 & 228.065 \\
\hline 3 & $C$ & $\mathrm{C}$ content & $\mathrm{wt} \%$ & {$[0.0006,0.495]$} & 0.054 & 0.049 \\
\hline 4 & $\mathrm{Si}$ & Si content & $\mathrm{wt} \%$ & {$[0.0001,1.151]$} & 0.052 & 0.106 \\
\hline 5 & $M n$ & Mn content & $\mathrm{wt} \%$ & {$[0.114,2.410]$} & 0.471 & 0.451 \\
\hline 6 & $\mathrm{Ni}$ & Ni content & $\mathrm{wt} \%$ & {$[0,0.075]$} & 0.009 & 0.007 \\
\hline 7 & $\mathrm{Cr}$ & Cr content & wt $\%$ & {$[0,0.547]$} & 0.031 & 0.077 \\
\hline 8 & $V$ & $\mathrm{~V}$ content & $\mathrm{wt} \%$ & {$[0,0.040]$} & 0.001 & 0.002 \\
\hline 9 & $\mathrm{Nb}$ & $\mathrm{Nb}$ content & $\mathrm{wt} \%$ & {$[0,0.056]$} & 0.004 & 0.010 \\
\hline 10 & Mo & Mo content & $\mathrm{wt} \%$ & {$[0,0.244]$} & 0.004 & 0.024 \\
\hline 11 & $\mathrm{Ti}$ & Ti content & $\mathrm{wt} \%$ & {$[0,0.174]$} & 0.021 & 0.029 \\
\hline 12 & $B$ & $\mathrm{~B}$ content & $\mathrm{wt} \%$ & {$[0,0.003]$} & 0.000 & 0.000 \\
\hline 13 & $\mathrm{Cu}$ & $\mathrm{Cu}$ content & $\mathrm{wt} \%$ & {$[0,0.273]$} & 0.014 & 0.035 \\
\hline 14 & $T_{w}$ & Water temperature & ${ }^{\circ} \mathrm{C}$ & {$[29.5,38.7]$} & 33.894 & 1.874 \\
\hline 15 & $P$ & Water pressure & Bar & {$[3,8.5]$} & 4.234 & 0.457 \\
\hline 16 & $T_{F}$ & Finish rolling delivery temperature & ${ }^{\circ} \mathrm{C}$ & {$[771,945]$} & 880.989 & 28.073 \\
\hline 17 & $V$ & Velocity & $\mathrm{m} / \mathrm{s}$ & {$[2.11,19.63]$} & 10.334 & 2.843 \\
\hline 18 & $F$ & Water distribution & - & - & - & - \\
\hline 19 & $q$ & Total flow & $\mathrm{m}^{3} / \mathrm{h}$ & {$[1880,12905]$} & 4615 & 1880 \\
\hline
\end{tabular}


Water distribution in each cooling zone represents cooling header opened/closed status and header water flow. Because of different cooling mechanism, UFC spray headers and laminar spray headers have different cooling efficiency even though in same cooling water flow. Moreover, water flow of UFC spray header can be adjusted in a wide range. In order to distinguish UFC header, laminar main cooling header and vernier header, cooling efficiency of $\boldsymbol{E}$ for different spray header is introduced. Taking one top header opened as example, heat transfer coefficient Eq. (6 7) can be transformed shown as followings Eq. (10). After cooling efficiency of different spray header are determined, water distribution in micro cooling zone can be indicatory as shown in Fig. 10. In addition, owing to the different number in each micro cooling zone, water distribution sequence is padding with 0 same with the closed spray headers.
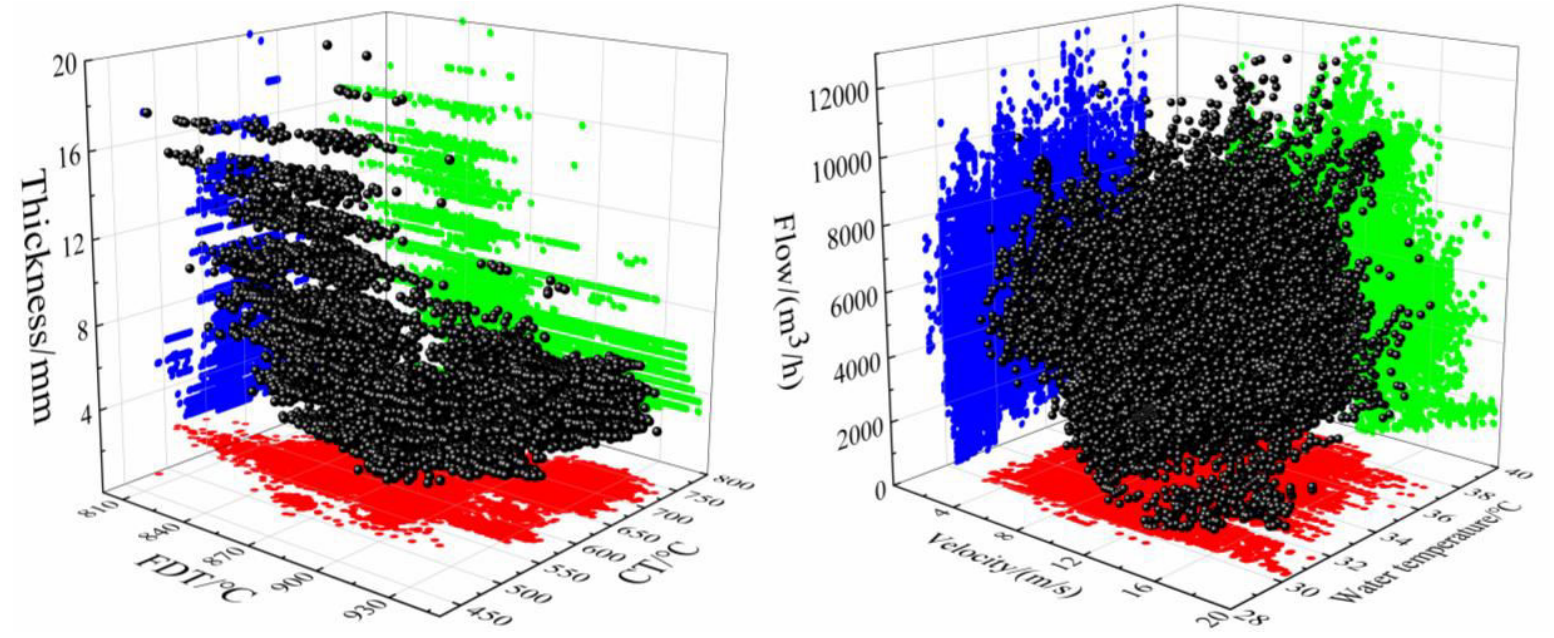

Fig. 9 Fractal dimension visualization diagrams of data

$$
h_{w}=E \cdot \frac{Q}{B L}(h)^{A_{2}} \cdot \exp \left(-A_{3}\left(t-T_{w}\right)\right)\left(\frac{T_{w}}{T_{o}}\right)^{A_{4}}\left(\frac{V}{V_{o}}\right)^{A_{G}}
$$


UFC: (01001 00101)

\begin{tabular}{|c|c|c|c|c|c|c|c|c|c|}
\hline 0 & 1.2 & 0 & 0 & 1.2 & 0 & 0 & 1.2 & 0 & 1.2 \\
\hline \multicolumn{10}{|c|}{ Main cooling: (1111) } \\
\hline 0 & 1 & 0 & 0 & 1 & 0 & 0 & 1 & 0 & 1 \\
\hline \multicolumn{10}{|c|}{ Trim cooling:(011100000) } \\
\hline 0 & 0.62 & 0.62 & 0.62 & 0.62 & 0 & 0 & 0 & 0 & 0 \\
\hline
\end{tabular}

Fig. 10 Schematic diagram of the water distribution padding with cooling efficiency in different micro cooling zone

\subsubsection{Industrial data processing}

In general, original data collected from hot rolling line always contains abnormal, noise and outliers, which may influence on modeling and lead to misleading predictions. Therefore, these data must be removed. In first, date of missing values was directly removed. And then, noise data was eliminated by Pauta criterion. The Pauta criterion implement in this paper was given as following Eqs. (11-13)[38], data with absolute deviation exceed the limitation will be regard as noise and removed. 18 key variables will be treated in this way.

$$
\begin{gathered}
\left|x_{i}-\mu\right|>3 \sigma \\
\mu=\frac{1}{n} \sum_{i}^{n} x_{i} \\
\sigma=\sqrt{\frac{1}{n} \sum_{i}^{n}\left(x_{i}-\mu\right)}
\end{gathered}
$$

where, $\mu$ is average of $x_{i}$ and $\sigma$ is standard deviation of samples. The result of abnormal detection with Pauta criterion is shown in Fig. 11. 


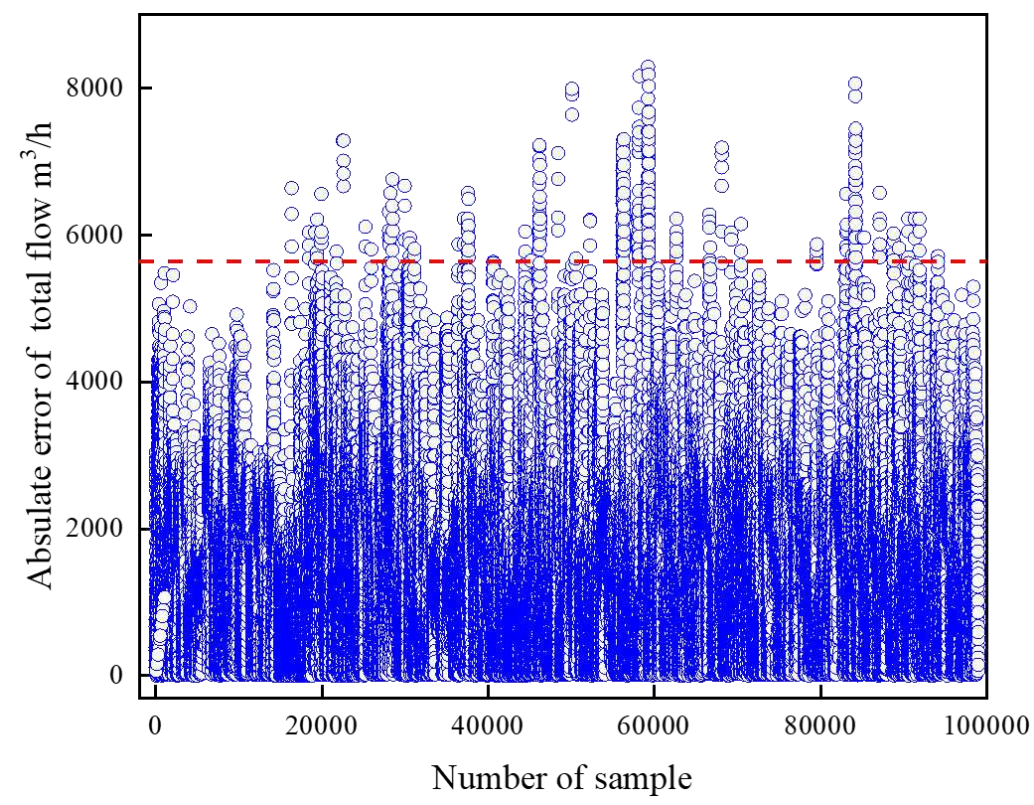

Fig. 11 Removing outliers with Pauta criterion

Due to Pauta criterion is only effective for normal distribution or approximate normal distribution, so in order to remove more outlier data, isolation forest is adopted to mining anomaly. Isolation forest algorithm, also named iforest, was proposed by Zhou[38]. Isolation forest is an efficient approach to anomaly detection that consider most points in data set are not anomalous, and anomalies are typically very different from the rest of dataset. Due to its linear time complexity and low demand for storage space, isolation forest has been used in many scenarios. This paper applies iforest to eliminate outlier of 19 features shown in Table 1. There are two hyperparameter isolation trees $t$ and sub-sampling size $\psi$ should be optimized for iforest. Herein, we use grid search method to select suitable for each parameter. The grid search result is shown in Fig. 12. As can be seen in Fig. 12, the fluctuating range of proportion is higher at small isolation tree $t$ and then stabilized when $t \geq 100$. In general, train process time will be linearly increasing with isolation and the iforest will be converged well when isolation tree $t=100$, the higher isolation has no meaning[38]. On the other hand, with the increasing of sub-sampling size $\psi$, the proportion is gradually decreasing, when $\psi$ is bigger than 256 , the proportion is stable in range of $0.14 \sim 0.16$. In summary, we select $t=100$ and $\psi=256$ as algorithm parameter. Base on low signal-to-noise ratio in general, 
top $2 \%$ rank in dataset was removed as abnormal data, after determined parameter.

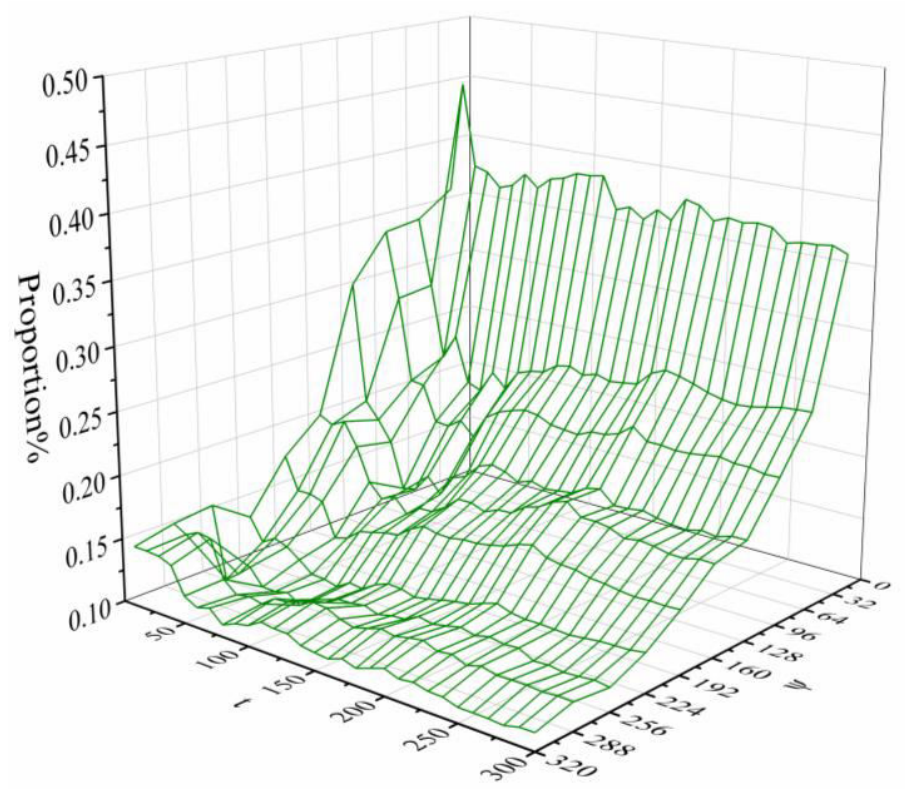

Fig. 12 The proportion of anomalies with the number of isolated trees and sub-sampling size

\subsubsection{Data normalization and division}

Different parameters often have different dimensions and units (as shown in Table 1). In order to eliminate the "eaten" situation caused by dimensions, input data must be normalized. The method used in this paper is zero-mean normalization and the formula is:

$$
x_{i}^{*}=\left(x_{i}-\mu\right) / \sigma
$$

where $x_{i}^{*}, x_{i}, \mu$ and $\sigma$ are normalized data, original data, mean of all the data and standard deviation.

After normalized, the dataset is split into training/validation/testing set with proportion $80 \%, 10 \%$ and $10 \%$, respectively.

Learning parameter depend on proposed model are trained with root mean square prop (RMSPorp) algorithm for minimizing error between prediction valve and actual value. The loss function of mean square error here is defined as:

$$
\text { Loss }=\frac{\sum_{i=1}^{n}\left(\hat{\mathrm{y}}_{i}-\mathrm{y}_{i}\right)^{2}}{N}
$$


where $\hat{\mathrm{y}}_{i}$ and $\mathrm{y}_{i}$ are prediction temperature distribution and actual temperature distribution in cooling zone, respectively, while $\mathrm{N}$ is the number of dataset. Due to only the start cooling temperature FDT and final CT in temperature distribution sequence could be measured, others are padded with traditional model recalculation values. If prediction error of temperature distribution were directly used as criterion of gradient descent, each prediction error of temperature in distribution sequence is equivalent, while measured actual value is worth specially utilized. Thus, an additional weight was added and the definition of new improved loss function is as following:

$$
\operatorname{Loss}=\frac{\sum_{j=1}^{N} w\left(\hat{\mathrm{y}}_{i}-\mathrm{y}_{i}\right)^{2}}{N}
$$

And weight of each error is derived from the distance with actual temperature measured as following Eq.

(17), the smaller distance between temperature distribution with actual temperature, the greater weight value of the loss corresponding.

$$
w_{j}=1 / \min (|j-n|,|j-1|)
$$

where, $j$ is serial number in sequence, $\mathrm{n}$ is length of the input sequence.

\section{Result and Discussion}

\subsection{Prediction of temperature distribution}

In this section, to build an optimal model, several experiments such as compassion concerning types of recurrent cells, sequence length and hyper-parameter settings are carried out and briefly illustrated. Above all, different types of recurrent cells are compared, i.e., standard RNN, LSTM and GRU. The recurrent cell shows a significant difference in extracting the temporal information via hidden states as introduced in $\mathbf{4 . 2}$. Secondly, the influence of length of input sequence, named timesteps was examined. Literatures show that vanishing gradient due to decay as sequence length increasing, makes the loss of earlier input sequence[38]. 
The performance of the proposed method is evaluated with prediction accuracy and computation time. Prediction accuracy is measured by three different metrics, root mean square error (RMSE), mean absolute error (MAE) and coefficient of determination $\left(\mathrm{R}^{2}\right)$. The formula of RMSE, MAE and $\mathrm{R}^{2}$ are as follows:

$$
\begin{array}{r}
R M S E=\frac{\sum_{i=1}^{N}\left(\hat{\mathrm{y}}_{i}-\mathrm{y}_{i}\right)}{N} \\
M A E=\frac{1}{N} \sum_{i=1}^{N}\left|\hat{\mathrm{y}}_{i}-\mathrm{y}_{i}\right| \\
R^{2}=1-\frac{\sum_{i=1}^{N}\left(y_{i}-\hat{y}_{i}\right)}{\sum_{i=1}^{N}\left(y_{i}-\bar{y}\right)^{2}}
\end{array}
$$

where $\hat{\mathrm{y}}_{i}$ and $\mathrm{y}_{i}$ are prediction and actual of temperature distribution in cooling zone, respectively, while $\mathrm{N}$ is number of the dataset. In addition, to evaluate whether it is competitive on the dynamic control for temperature distribution prediction in control cooling process, computation time of proposed method is compared with traditional model.

The evaluation criteria result of prediction accuracy were as depicted in Table 2. As can be seen in Table 2, Models based on LSTM and GRU were slightly outperform than ones with standard RNN. The models based on bidirectional unit shown similar performance regularity, because bidirectional unit performs in sequential dependencies of forwarding and backward direction [39], it is observed that the bidirectional unit of each model generally enhances the prediction accuracy. In addition, it is shown that length of input sequence (timesteps) affects prediction accuracy of each recurrent unit. Due to higher velocity prediction accuracy in more timesteps, the models with 48 timesteps have higher prediction performance in both unidirectional and bidirectional models, even if long sequence preventing from capturing a piece of extended temporal[40]. As forementioned, the more timesteps means the more times of velocity modify calculation, that higher accuracy velocity prediction will be realized, which also makes the proposed model yield higher performance. Conclusion, the bi-LSTM model yielded the better value for 
each evaluation criterion than other units, and the model based on bi-LSTM and 48 timesteps showed best prediction performance with lowest RMSE 8.03 and MAE 5.7, highest $\mathrm{R}^{2} 0.976$.

Table 2. Performance comparison of proposed model with different recurrent cell and timestep

\begin{tabular}{c|ccc|ccc|ccc}
\hline \multirow{2}{*}{ Recurrent cell } & \multicolumn{3}{|c|}{ RMSE } & \multicolumn{3}{c|}{ MAE } & \multicolumn{3}{c}{$\mathrm{R}^{2}$} \\
\cline { 2 - 11 } & 12 & 24 & 48 & 12 & 24 & 48 & 12 & 24 & 48 \\
\hline \multirow{2}{*}{\begin{tabular}{c} 
RNN \\
bi-RNN \\
LSTM \\
bi-LSTM \\
\cline { 2 - 11 }
\end{tabular}} & 22.2 & 17.3 & 14.9 & 17.6 & 12.8 & 10.6 & 0.819 & 0.890 & 0.918 \\
\cline { 2 - 10 } GRU & 15.6 & 9.8 & 8.78 & 11.4 & 7.2 & 6.1 & 0.911 & 0.964 & 0.971 \\
\cline { 2 - 10 } bi-GRU & $\mathbf{1 4 . 2}$ & $\mathbf{9 . 4 3}$ & $\mathbf{8 . 0 3}$ & $\mathbf{1 0 . 4}$ & $\mathbf{6 . 7}$ & $\mathbf{5 . 7}$ & $\mathbf{0 . 9 2 5}$ & $\mathbf{0 . 9 6 7}$ & $\mathbf{0 . 9 7 6}$ \\
\cline { 2 - 10 } & 17.2 & 15.4 & 12.8 & 12.6 & 10.1 & 9.1 & 0.889 & 0.891 & 0.939 \\
\cline { 2 - 10 } & 14.5 & 10.7 & 8.29 & 10.4 & 7.8 & 5.9 & 0.922 & 0.943 & 0.957 \\
\hline
\end{tabular}

In order to further evaluate performance of proposed model for temperature distribution prediction, several segment with different rolling velocity of $11.8 \mathrm{~mm} 380 \mathrm{CL}$ strip were selected as examples, temperature distribution prediction were described in Fig. 13. Compared with baseline model, the proposed model with 48 timesteps yielded desirable prediction as shown in Fig. 13, which proved the ability of temperature distribution prediction. Moreover, the final CT prediction performance of models based on biLSTM were shown in Fig. 14. It can be clearly seen that temperature prediction error decreased with increasing of input length. And prediction accuracy within $\pm 20^{\circ} \mathrm{C}$ were $94.9 \%, 95.6 \%$ and $97.6 \%$ for 12,24 , 48 timesteps, respectively, equaling or higher than the of baseline model, whose best accuracy within $\pm 20^{\circ} \mathrm{C}$ is $95 \%$ in application. Furthermore, the model based on bi-LSTM and 48 timesteps could realize $95 \%$ prediction accuracy of $\pm 15^{\circ} \mathrm{C}$, meaning more times of velocity modifying is advantage for temperature prediction and control. 

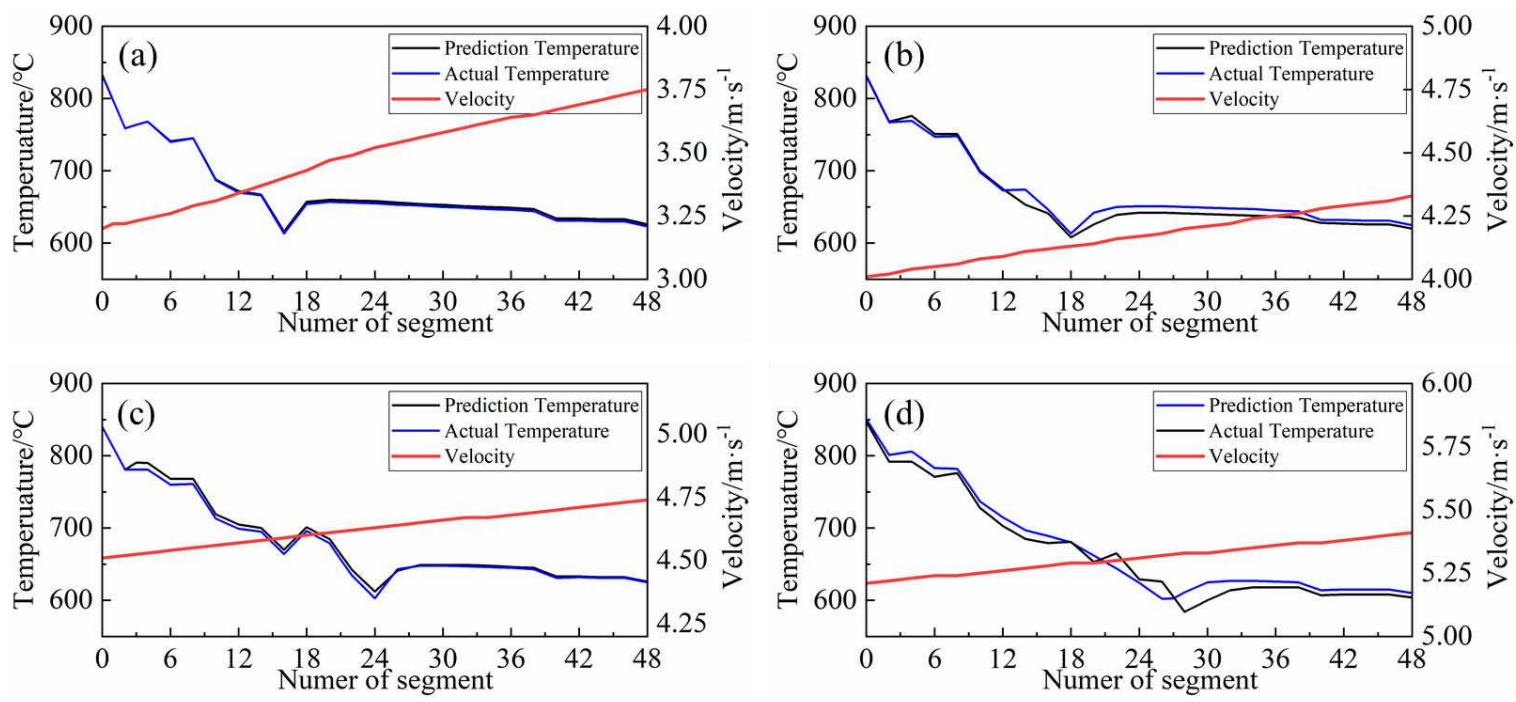

Fig. 13 Prediction and measured temperature distribution of $11.8 \mathrm{~mm}$ 380CL under rolling stage
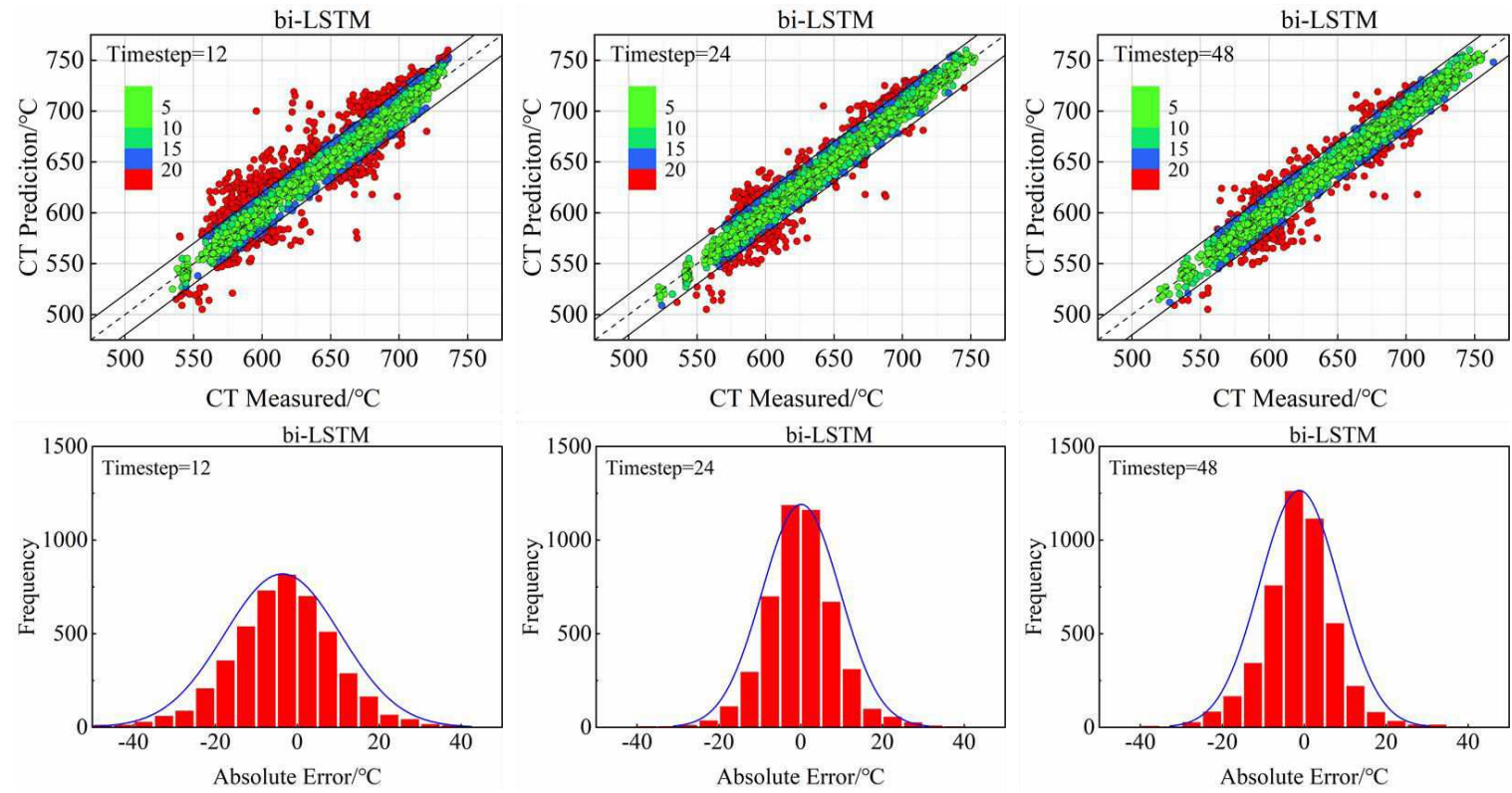

Fig. 14. Regression results and absolute error histograms with bi-LSTM and 48 timesteps

\subsection{Computation time}

We aim to develop not only a high precision prediction but also a real-time model which could just in-

time deal with variable-velocity rolling. Thus, the model should deal with variable-velocity dynamic process and satisfy the requirement of online temperature monitor and modify. Herein, proposed deep neural network-based model and traditional mechanism model were built to compared to computation time.

Table 3 showed the comparison result for each model with different timesteps. It is shown that 
computational cost of the proposed model is considerably lower than baseline mechanism model. Baseline mechanism model requires excessive computational resources by 1.2, 1.7 and 2.5 seconds for 12,24 and 48 timesteps to accomplish re-calculation of temperature distribution for all segment in cooling process, respectively. While, the proposed model based on bi-LSTM show relatively similar consume time less than 0.001 second.

Table 3 Comparison of the computation time between the mechanism model and proposed modell

\begin{tabular}{lccc}
\hline Model & $\begin{array}{c}12 \text { timesteps } \\
\text { second }\end{array}$ & $\begin{array}{c}24 \text { timesteps } \\
\text { second }\end{array}$ & $\begin{array}{c}48 \text { timesteps } \\
\text { second }\end{array}$ \\
\hline Proposed model & 0.0009 & 0.0009 & 0.0008 \\
Mechanism model & 1.2 & 1.7 & 2.5 \\
\hline
\end{tabular}

To summarize, the proposed model outperformed mechanism model concerning computation time, which makes it possible to establish a real-time dynamic control in an actual control cooling process.

\section{Conclusions and Future Work}

In this paper, a temperature distribution prediction based on recurrent neural network for variablevelocity hot rolling strips was proposed. The isolated forest algorithm was used to eliminate outliers. And different recurrent cell and length of input sequence were tested to determined optimal model. The key results were as follows:

(1) A traditional mechanism model of temperature distribution prediction based on finite-difference method was introduced as baseline model. Moreover, to match precise velocity value, an online cycle velocity calculation strategy was proposed. The result showed that, the proposed velocity calculation strategy could realize high precise velocity prediction.

(2) The Pauta criterion and isolate forest algorithm were used to eliminate outliers, successively. Loss function was modified to make it more suitable for the training the proposed model, due to measured 
temperature should occupy more important influence.

(3) The prediction performance with standard RNN, LSTM, GRU and bidirectional operation recurrent cell and length of input sequence of 12, 24 and 48 were compared with evaluation criteria of MSE, MAE and $\mathrm{R}^{2}$. Among them, the model built with bi-LSTM and 48 timesteps yielded best performance in terms of prediction accuracy, and has lowest RMSE 8.03 and MAE 5.7, highest $\mathrm{R}^{2} 0.976$.

(4) Compared with baseline model, the proposed model cost fewer times (less than 0.001 seconds for one cycle temperature distribution prediction of all segment in cooling zone), which increases feasibility of model used online for industrial application.

It is feasible to unitize RNN model to realize temperature distribution prediction for hot strip control cooling process on run-out table. The follow-up work is going to improve prediction accuracy by obtaining more input features and testing more effective model structures, such as convolutional neural network + recurrent neural network, which is beneficial for recognizing more information from sequence data. In addition, the input feature of water distribution should be considered for influence effect on cooling, and influence effect should be recognized by the proposed model to improve the prediction performance.

\section{Authors' contribution}

Dong Chen: Methodology, Data curation, Visualization, Investigation, Writing - original draft. Rui Zhang

Data curation, Software, Visualization. Zhenlei Li: Conceptualization, Methodology, Supervision, Validation, Writing - review \& editing. Yunjie Li: Visualization. Guo Yuan: Conceptualization, Supervision, Validation, Writing - review \& editing 


\section{Fundings}

This work was supported by the National Natural Science Foundation of China (51804074), Project funded by China Postdoctoral Science Foundation (2020M680964), Fundamental Research Funds for the Central Universities (N2107004) and Northeastern University Postdoctoral Foundation (20200323).

\section{Availability of data and materials}

All the data that supports the findings of study is available from the corresponding author upon reasonable request.

\section{Declarations}

We promise that our research in this work is in compliance with ethical standards all the while.

Competing interests The authors declare no competing interests.

Consent to participate All the authors consent to participate in this work.

Consent to publication The authors agree to publication in the Journal indicated below and also to publication of article in English by Springer in Springr's corresponding English-language journal.

\section{References}

[1] Herman JC (2001) Impact of new rolling and cooling technologies on thermomechanically processed steels. Ironmak Steelmak 28:159-63. https://doi.org/10.1179/030192301677894.

[2] Tsuyama S (2015) Thick plate technology for the last 100 years: A world leader in Thermo Mechanical Control Process. ISIJ Int 55:67-78. https://doi.org/10.2355/isijinternational.55.67.

[3] Uranga P, Rodríguez-Ibabe JM (2020). Thermomechanical processing of steels. Metals (Basel) 10. https://doi.org/10.3390/met10050641.

[4] Li C, Yuan G, Ji Fet al (2015) Mechanism of microstructural control and mechanical properties in hot 
rolled plain C-Mn steel during controlled cooling. ISIJ Int 55:1721-9.

https://doi.org/10.2355/isijinternational.ISIJINT-2015-040.

[5] Shi J, Yuan G, Jiang L et al (2015). Heat Transfer Symmetry of the Strip Surface Due to Agroup Oblique Slot Jet Impingement after Hot Rolling. Steel Res Int 86:1548-57. https://doi.org/10.1002/srin.201400529.

[6] Timm W, Weinzierl K, Leipertz A, et al (2002) Modelling of heat transfer in hot strip mill runout table cooling. Steel Res 73:97-104. https://doi.org/10.1002/srin.200200180.

[7] Li HJ, Li ZL, Yuan G et al (2013) Development of new generation cooling control system after rolling in hot rolled strip based on UFC. J Iron Steel Res Int 20:29-34. https://doi.org/10.1016/S1006-706X(13)60122-3. [8] Gong DY, Xu JZ, Cai XH et al (2007) Model Building of Finishing Temperature Control for Accelerative Hot Strip Rolling. Key Eng Mater 340-341:633-8. https://doi.org/10.4028/www.scientific.net/kem.340-341.633.

[9] Xie HB, Jiang ZY, Liu XH et al (2007) On-Line Optimization of Coiling Temperature Control on Run-Out Table for Hot Strip Mills. Key Eng Mater 340-341:701-6. https://doi.org/10.4028/www.scientific.net/kem.340341.701.

[10] Latzel S (2001). Advanced automation concept of runout table strip cooling for hot strip and plate mills.

IEEE Trans Ind Appl 37:1088-97. https://doi.org/10.1109/28.936401.

[11] Yu QB, Wang ZD, Wang ZY (2003) Improvement of Prediction Method for Strip Coiling Temperature. J Iron Steel Res Int;10:p.75-78.

[12] Zheng Y, Li N, Li S (2013) Hot-rolled strip laminar cooling process plant-wide temperature monitoring and control. Control Eng Pract 21:23-30. https://doi.org/10.1016/j.conengprac.2012.09.004.

[13] Xie HB, Liu XH, Wang GD et al (2006) Optimization and model of laminar cooling control system for hot strip mills. J Iron Steel Res Int 13:18-22. https://doi.org/10.1016/S1006-706X(06)60019-8.

[14] Li S, Li X, Yang Y (2016) Intelligent model building and GPC-PID based temperature curve control 
strategy for metallurgical industry. Math Probl Eng. https://doi.org/10.1155/2016/7454805.

[15] Li S, Zhou C, Deng Z et al (2015) Application of genetic algorithm and least squares support vector machines in laminar cooling process. J Comput Theor Nanosci 12:1467-71.

https://doi.org/10.1166/jctn.2015.3915.

[16] Li S, Li X, Deng Z (2014) A new kind of model of laminar cooling: By ls-svm and genetic algorithm.

Commun Comput Inf Sci 472:251-4. https://doi.org/10.1007/978-3-662-45049-9_41.

[17] Li S, Li X, Deng Z (2016) A T-S fuzzy model-based intelligent temperature prediction model of laminar cooling system. Proc-2015 Chinese Autom Congr CAC 2015 1221-4.

https://doi.org/10.1109/CAC.2015.7382685.

[18] Zheng Y, Li S, Wang X (2011) Horizon-varying model predictive control for accelerated and controlled cooling process. IEEE Trans Ind Electron 58:329-36. https://doi.org/10.1109/TIE.2010.2045321.

[19] Zheng Y, Li S, Wang X (2009) Distributed model predictive control for plant-wide hot-rolled strip laminar cooling process. J Process Control 19:1427-37. https://doi.org/10.1016/j.jprocont.2009.04.012.

[20] Li HJ, Li LG, Li YL et al (2015) Online Monitor and Control of Cooling Temperature on Run-out Table of Hot Strip Mill. Steel Res Int 86:1225-33. https://doi.org/10.1002/srin.201400269.

[21] Chen XL, Wang GD, Tian Y et al (2014) An On-line Finite Element Temperature Field Model for Plate Ultra Fast Cooling Process. J Iron Steel Res Int 21:481-7. https://doi.org/10.1016/S1006-706X(14)60075-3.

[22] Li ZL, Li HJ, Yuan G et al (2015) Research and application of ultra-fast cooling system and velocity controlled strategy for hot rolled strip. Steel Res Int 86:478-88. https://doi.org/10.1002/srin.201400077. [23] Zheng Y, Li S, Wang X (2010) An approach to model building for accelerated cooling process using instance-based learning. Expert Syst Appl 37:5364-71. https://doi.org/10.1016/j.eswa.2010.01.020.

[24] Zhang T, Xie Q, Wang B et al (2016) A Novel Variable Scale Grid Model for Temperature Self-Adaptive 
Control: An Application on Plate Cooling Process after Rolling. Steel Res Int 87:1213-9.

https://doi.org/10.1002/srin.201500344.

[25] Schlang M, Broese E, Feldkeller B et al (1997). Neural networks for process control in steel manufacturing.

ICASSP, IEEE Int Conf Acoust Speech Signal Process - Proc1:155-8.

https://doi.org/10.1109/icassp.1997.599582.

[26] Liu EY, Peng W, Cao N et al (2014) Prediction of coiling temperature of hot rolled strip based on BP

neural network. Appl Mech Mater 633-634:679-83. https://doi.org/10.4028/www.scientific.net/AMM.633-

634.679.

[27] Deng J, Sun J, Peng W et al (2019) Application of neural networks for predicting hot-rolled strip crown.

Appl Soft Comput J 78:119-31. https://doi.org/10.1016/j.asoc.2019.02.030.

[28] Colla V, Vannucci M, Dimatteo A (2010) Diagnosis of the instability of the cooling behaviour of flat steel products through parametric characterisation, neural networks and statistics. ISA Trans 49:235-43.

https://doi.org/10.1016/j.isatra.2010.01.001.

[29] Xing G, Ding J, Chai T et al (2012) Hybrid intelligent parameter estimation based on grey case-based reasoning for laminar cooling process. Eng Appl Artif Intell 25:418-29.

https://doi.org/10.1016/j.engappai.2011.10.007.

[30] Rumelhart DE, Hintont GE (1986) Learning Representations by Back-Propagating Errors. Cogn Model:3-6. https://doi.org/10.7551/mitpress/1888.003.0013.

[31] Connor JT, Martin RD, Atlas LE (1994) Recurrent Neural Networks and Robust Time Series Prediction.

IEEE Trans Neural Networks 5:240-54. https://doi.org/10.1109/72.279188.

[32] Gregor K, Danihelka I, Graves A et al (2015) A recurrent neural network for image generation. 32nd Int Conf Mach Learn ICML 2:1462-71. 
[33] Alex Graves AM and GH (2013) Speech Recognition with Deep Recurrent Neural Networks, Department of Computer Science, University of Toronto. Dep Comput Sci Univ Toronto 3:45-9.

[34] Sak H, Senior A, Beaufays F ( 2014) Long Short-Term Memory Based Recurrent Neural Network Architectures for Large Vocabulary Speech Recognition.

[35] Graves A, Schmidhuber J (2005) Framewise phoneme classification with bidirectional LSTM and other neural network architectures. Neural Networks 18:602-10. https://doi.org/10.1016/j.neunet.2005.06.042.

[36] Heck JC, Salem FM (2017) Simplified minimal gated unit variations for recurrent neural networks. Midwest Symp Circuits Syst 2017-Augus:1593-6. https://doi.org/10.1109/MWSCAS.2017.8053242.

[37] Dey R, Salemt FM (2017). Gate-variants of Gated Recurrent Unit (GRU) neural networks. arXiv. https://doi.org/10.1109/MWSCAS.2017.8053243.

[38] Li L, Wen Z, Wang Z (2016) Outlier detection and correction during the process of groundwater lever monitoring base on pauta criterion with self-learning and smooth processing. Commun Comput Inf Sci 643:497503. https://doi.org/10.1007/978-981-10-2663-8_51.

[39] Thireou T, Reczko M (2007) Bidirectional long short-term memory networks for predicting the subcellular localization of eukaryotic proteins. IEEE/ACM Trans Comput Biol Bioinforma 4:441-6. https://doi.org/10.1109/TCBB.2007.1015.

[40] Lee SY, Tama BA, Choi C et al (2020) Spatial and Sequential Deep Learning Approach for Predicting Temperature Distribution in a Steel-Making Continuous Casting Process. IEEE Access 8:21953-65. https://doi.org/10.1109/ACCESS.2020.2969498. 